\title{
Spectral Reflectance Estimation and Color Reproduction Based on Sparse Neugebauer Model
}

\author{
Kohei Inoue*, Kenji Hara, Kiichi Urahama \\ Department of Communication Design Science, Faculty of Design, Kyushu University, 815-8540, Japan
}

\begin{tabular}{l} 
A R T I C L E I N F O \\
\hline Article history: \\
Received: 29 April, 2017 \\
Accepted: 13 June, 2017 \\
Online: 30 June, 2017 \\
\hline Keywords: \\
Spectral Reflectance \\
Color Reproduction \\
Sparse Neugebauer Model \\
\hline
\end{tabular}

\section{Introduction}

The reflectance properties of object surfaces are determined by the spectral reflectance factors that are normally measured at regular intervals in the visible spectrum of radiation by reflectance spectrophotometers [1]. The integrals of the products of the spectral reflectance, the relative spectral power distribution of an illuminant and color matching functions produce the tristimulus values. On the other hand, the inverse computation from tristimulus values or colorimetric data to the spectral reflectance is a difficult problem because the computation of tristimulus values from spectral reflectance involves the dimensionality reduction of data, i.e., the spectral reflectance estimation is an ill-posed problem.

Recently, Sharma and Wang proposed a neural network-based spectrum recovery method, and evaluated the accuracy of the spectrum recovery by the spectral mean-squared error [2]. Harifi et al. presented a principal component analysis (PCA)-based method which employed six principal components [3]. Amiri and Amirshahi proposed a step by step progressive method for recovery of spectral data from the corresponding colorimetric information, where the spatial dimensions are decreased from 6D to $3 \mathrm{D}$ in a step-by-step manner to decrease the number of samples used for color gamut interpolation [4]. Wu et al. presented an approach that uses a new sample selection criterion based on color "Corresponding Author: Kohei Inoue, Department of Communication Design Science, Faculty of Design, Kyushu University, Japan.

Email: k-inoue@design.kyushu-u.ac.jp feature match to select a series of suitable samples for creating the adapted transformation matrix to reconstruct spectral reflectance [5]. Inoue et al. proposed two methods for recovering the reflectance spectra of colorimetric data by using the non-negative constraints in reflectance spectra, where the problem of reflectance spectra recovery is formulated as a nonnegative least squares problem [6] into which the Neugebauer model [7] is incorporated [8]. These methods can be used to estimate the spectral reflectance from general colorimetric data including CIE tristimulus values and CMYK-printed samples.

The Neugebauer model can be used to infer the gamut boundaries of printed colorants on paper [7] by printers such as conventional CMYK printers. If three inks, such as cyan, magenta and yellow, are utilized, then the paper may be covered by one ink, two inks, three inks, or may be left blank. As a result, eight combinations of overlap of inks can occur, and they correspond to eight basic colors, such as red, green, blue, black, cyan, magenta, yellow and white, which are referred to as the Neugebauer primaries [7]. Although the Neugebauer model was originally developed for modeling the printed colorants on paper, it can be used for general colorimetric data as we will prove in Subsection 2.1 that the equation of the Neugebauer model holds for any color in RGB color space.

In this paper, we propose sparse Neugebauer model for estimating spectral reflectance of Neugebauer primaries or basic eight colors when reflectance spectra of several colors are given. Experimental results show that the proposed method achieves 
lower estimation error than the previous method with the conventional Neugebauer model. We also reproduce the Neugebauer primaries and the colors of given reflectance spectra from the estimated reflectance spectra. The effectiveness of the sparse Neugebauer model in color reproduction is also demonstrated in comparison with the conventional Neugebauer model.

The rest of this paper is organized as follows. Section 2 summarizes the Neugebauer model, and then proposes the sparse Neugebauer model. Section 3 proposes a method for estimating the spectral reflectance based on the Neugebauer models, and a convergence property of the proposed estimation algorithm is proved. Section 4 describes the procedure for reproducing the colors from the estimated spectral data. Section 5 shows experimental results. Finally, Section 6 concludes this paper.

\section{Neugebauer Models}

In this section, we first summarize the Neugebauer model, and then propose sparse Neugebauer model to improve the performance of spectral reflectance estimation and color reproduction.

\subsection{Neugebauer Model}

Let $\boldsymbol{v}=[r, g, b]$ be a color vector in RGB color space (bold italic lowercase letters denote vectors), where $r, g$ and $b$ denote the red, green and blue components of the color, and satisfy $0 \leq$ $r \leq 1,0 \leq g \leq 1$ and $0 \leq b \leq 1$, respectively. Then $v$ have another expression as follows:

$$
\boldsymbol{v}=a_{1} \boldsymbol{r}+a_{2} \boldsymbol{g}+a_{3} \boldsymbol{b}+a_{4} \boldsymbol{k}+a_{5} \boldsymbol{c}+a_{6} \boldsymbol{m}+a_{7} \boldsymbol{y}+a_{8} \boldsymbol{w}
$$

where $\quad \boldsymbol{r}=[1,0,0], \boldsymbol{g}=[0,1,0], \boldsymbol{b}=[0,0,1], \boldsymbol{k}=[0,0,0], \boldsymbol{c}=$ $\boldsymbol{w}-\boldsymbol{r}, \boldsymbol{m}=\boldsymbol{w}-\boldsymbol{g}, \boldsymbol{y}=\boldsymbol{w}-\boldsymbol{b}$ and $\boldsymbol{w}=[1,1,1]$ denote the Neugebauer primaries [7]: red, green, blue, black, cyan, magenta, yellow and white, respectively. The coefficients in (1) are given by

$$
\begin{aligned}
& a_{1}=r(1-g)(1-b), a_{5}=(1-r) g b, \\
& a_{2}=(1-r) g(1-b), a_{6}=r(1-g) b, \\
& a_{3}=(1-r)(1-g) b, a_{7}=r g(1-b), \\
& a_{4}=(1-r)(1-g)(1-b), a_{8}=r g b,
\end{aligned}
$$

which are called the Demichel equations, and (1) is called the Neugebauer equation [7]. The coefficients in (2) are nonnegative and satisfy $\sum_{k=1}^{K} a_{k}=1$ where $K=8$. The equality in (1) with the coefficients in (2) can be confirmed for each element as follows: for example, the first element in (1) satisfies

$$
\begin{gathered}
r(1-g)(1-b)+r(1-g) b+r g(1-b)+r g b \\
=r[(1-g)(1-b)+(1-g) b+g(1-b)+g b] \\
=r[(1-g)\{(1-b)+b\}+g\{(1-b)+b\}] \\
=r[(1-g)+g][(1-b)+b]=r .
\end{gathered}
$$

The similar equations hold for the second and third elements in (1).
We simply call (1) with (2) the Neugebauer model, which is valid for both tristimulus values and spectral reflectance, and use it for spectral reflectance estimation.

\subsection{Sparse Neugebauer Model}

Let $S=\{\boldsymbol{r}, \boldsymbol{g}, \boldsymbol{b}, \boldsymbol{k}, \boldsymbol{c}, \boldsymbol{m}, \boldsymbol{y}, \boldsymbol{w}\}$ be a set of the Neugebauer primaries. Then the Neugebauer equation in (1) states that an arbitrary color vector $\boldsymbol{v}$ can be expressed as a weighted average of the eight color vectors in $S$. We can also express a color vector in RGB color space as a linear combination of four colors which are in general position in RGB color space because four points being in general position in a three-dimensional color span a threedimensional linear space, and form a tetrahedron. If a color is just on a plane spanned by three vertices of RGB color cube or a line connecting two vertices of RGB color cube, then the color is expressed as the linear combinations of three or two vertices, respectively. We describe the most general four-color case as follows (in the other degenerated cases, the description can be simplified): Let $\tilde{S}=\left\{\boldsymbol{s}_{1}, \boldsymbol{s}_{2}, \boldsymbol{s}_{3}, \boldsymbol{s}_{4}\right\}$ be a subset of $S$ the elements of which are in general position. Then, for a color vector $\boldsymbol{v}$, we have

$$
\begin{gathered}
\boldsymbol{v}-\boldsymbol{s}_{1}=\alpha_{2}\left(\boldsymbol{s}_{2}-\boldsymbol{s}_{1}\right)+\alpha_{3}\left(\boldsymbol{s}_{3}-\boldsymbol{s}_{1}\right)+\alpha_{4}\left(\boldsymbol{s}_{4}-\boldsymbol{s}_{1}\right) \\
=\left[\alpha_{2}, \alpha_{3}, \alpha_{4}\right]\left[\begin{array}{l}
\boldsymbol{s}_{2}-\boldsymbol{s}_{1} \\
\boldsymbol{s}_{3}-\boldsymbol{s}_{1} \\
\boldsymbol{s}_{4}-\boldsymbol{s}_{1}
\end{array}\right]
\end{gathered}
$$

from which the coefficients $\alpha_{2}, \alpha_{3}, \alpha_{4}$ are given by

$$
\left[\alpha_{2}, \alpha_{3}, \alpha_{4}\right]=\left(\boldsymbol{v}-\boldsymbol{s}_{1}\right)\left[\begin{array}{l}
\boldsymbol{s}_{2}-\boldsymbol{s}_{1} \\
\boldsymbol{s}_{3}-\boldsymbol{s}_{1} \\
\boldsymbol{s}_{4}-\boldsymbol{s}_{1}
\end{array}\right]^{-1}
$$

where $\boldsymbol{s}_{1}$ is selected as a basic point. As a result, we have

$$
\boldsymbol{v}=\alpha_{1} \boldsymbol{s}_{1}+\alpha_{2} \boldsymbol{s}_{2}+\alpha_{3} \boldsymbol{s}_{3}+\alpha_{4} \boldsymbol{s}_{4}
$$

where $\alpha_{1}=1-\alpha_{2}-\alpha_{3}-\alpha_{4}$, and the four coefficients are nonnegative if $\boldsymbol{v}$ is in the tetrahedron with the four vertices in $\tilde{S}$. We call (3) the sparse Neugebauer model since four coefficients among eight ones in (1) are zero in (3).

We adapt the minimal brightness variation criterion (MBVQ) proposed by Shaked et al. [9] for selecting four Neugebauer primaries $\boldsymbol{s}_{1}, \boldsymbol{s}_{2}, \boldsymbol{s}_{3}, \boldsymbol{s}_{4}$, which are called the minimal brightness variation quadruple (MBVQ) [9], and given by $\{\boldsymbol{r}, \boldsymbol{g}, \boldsymbol{b}, \boldsymbol{k}\},\{\boldsymbol{w}, \boldsymbol{c}, \boldsymbol{m}, \boldsymbol{y}\},\{\boldsymbol{m}, \boldsymbol{y}, \boldsymbol{g}, \boldsymbol{c}\},\{\boldsymbol{r}, \boldsymbol{g}, \boldsymbol{m}, \boldsymbol{y}\},\{\boldsymbol{r}, \boldsymbol{g}, \boldsymbol{b}, \boldsymbol{m}\}$ or $\{\boldsymbol{c}, \boldsymbol{m}, \boldsymbol{g}, \boldsymbol{b}\}$.

\section{Spectral Reflectance Estimation}

Assume that the reflectance spectra of $m$ colors $\boldsymbol{v}_{i}=$ $\left[r_{i}, g_{i}, b_{i}\right]$ for $i=1, \ldots, m$ are given and stored in $Q=\left[q_{i j}\right]$, where $q_{i j}$ denotes the reflectance of the $j$ th wavelength $\lambda_{j}$ for $j=$ $1, \ldots, n$ in the $i$ th color $v_{i}$. Then, by the above nonsparse or sparse Neugebauer model, we have

$$
Q \approx A P
$$

where $A=\left[a_{i k}\right]$ denotes an $m \times K$ coefficient matrix whose elements are given by 


$$
\begin{aligned}
& a_{i, 1}=r_{i}\left(1-g_{i}\right)\left(1-b_{i}\right), \quad a_{i, 5}=\left(1-r_{i}\right) g_{i} b_{i}, \\
& a_{i, 2}=\left(1-r_{i}\right) g_{i}\left(1-b_{i}\right), \quad a_{i, 6}=r_{i}\left(1-g_{i}\right) b_{i}, \\
& a_{i, 3}=\left(1-r_{i}\right)\left(1-g_{i}\right) b_{i}, \quad a_{i, 7}=r_{i} g_{i}\left(1-b_{i}\right), \\
& a_{i, 4}=\left(1-r_{i}\right)\left(1-g_{i}\right)\left(1-b_{i}\right), \quad a_{i, 8}=r_{i} g_{i} b_{i},
\end{aligned}
$$

when the Neugebauer model is used, and these are the Demichel equations [7] for the $i$ th color $\boldsymbol{v}_{i}$, and the number of columns in $A$ is the number of the Neugebauer primaries, i.e., $K=8$. For the sparse Neugebauer model, four coefficients out of the eight ones in (4) become zero. $P=\left[p_{k j}\right]$ denotes a $K \times n$ matrix whose $(k, j)$ element $p_{k j}$ is the reflectance of the $k$ th Neugebauer primary at the $j$ th wavelength $\lambda_{j}$. Then we formulate the problem for estimating the spectral reflectance of the Neugebauer primaries as follows:

$$
\begin{aligned}
& \min _{P}\|Q-A P\|_{F}^{2}, \\
& \text { subj. to } p_{k j} \geq 0,
\end{aligned}
$$

where $\|\cdot\|_{F}$ denotes the Frobenius norm, and the constraint (6) is for $k=1, \ldots, K$ and $j=1, \ldots, n$. Let $E(P)$ be the objective function in (5), then we have

$$
\begin{gathered}
E(P)=\sum_{i=1}^{m} \sum_{j=1}^{n}\left(q_{i j}-\sum_{k=1}^{K} a_{i k} p_{k j}\right)^{2} \\
=\sum_{i=1}^{m} \sum_{j=1}^{n}\left[q_{i j}^{2}-2 q_{i j} \sum_{k=1}^{K} a_{i k} p_{k j}+\left(\sum_{k=1}^{K} a_{i k} p_{k j}\right)^{2}\right] .
\end{gathered}
$$

Since $\sum_{i=1}^{m} \sum_{j=1}^{n} q_{i j}^{2}$ is a constant independent of $p_{k j}$, the minimization of $E(P)$ is equivalent to the maximization of

$$
F(P)=\sum_{i=1}^{m} \sum_{j=1}^{n}\left[q_{i j} \sum_{k=1}^{K} a_{i k} p_{k j}-\frac{1}{2}\left(\sum_{k=1}^{K} a_{i k} p_{k j}\right)^{2}\right] .(7)
$$

Applying Jensen's inequality [10] to the second term of the right side in (7), we have

$$
\begin{aligned}
& \left(\sum_{k=1}^{K} a_{i k} p_{k j}\right)^{2}=\left(\sum_{k=1}^{K} u_{i j k} \frac{a_{i k} p_{k j}}{u_{i j k}}\right)^{2} \\
& \leq \sum_{k=1}^{K} u_{i j k}\left(\frac{a_{i k} p_{k j}}{u_{i j k}}\right)^{2}=\sum_{k=1}^{K} \frac{\left(a_{i k} p_{k j}\right)^{2}}{u_{i j k}},
\end{aligned}
$$

where $u_{i j k}$ are positive constants satisfying $\sum_{k=1}^{K} u_{i j k}=1$. Substituting the rightmost term in (8) into (7), we have a lower bound of $F(P)$ as

$$
F_{L B}(P, U)=\sum_{i=1}^{m} \sum_{j=1}^{n}\left[q_{i j} \sum_{k=1}^{K} a_{i k} p_{k j}-\frac{1}{2} \sum_{k=1}^{K} \frac{\left(a_{i k} p_{k j}\right)^{2}}{u_{i j k}}\right] \text {,(9) }
$$

where $U=\left[u_{i j k}\right]$ denotes a three-dimensional array having $u_{i j k}$ as its $(i, j, k)$ element.

Replacing $F$ with $F_{L B}$, we formulate the problem of spectral reflectance estimation as follows:

$$
\begin{gathered}
\max _{P, U} F_{L B}(P, U) \\
\text { subj. to } \quad \sum_{k=1}^{K} u_{i j k}=1, u_{i j k}>0, \quad p_{k j} \geq 0 .
\end{gathered}
$$

Let $L=F_{L B}-\sum_{i=1}^{m} \sum_{j=1}^{n} \mu_{i j}\left(\sum_{k=1}^{K} u_{i j k}-1\right)$ be the Lagrange function for the constrained maximization problem (10) with (11), where $\mu_{i j}$ for $i=1, \ldots, m$ and $j=1, \ldots, n$ are the Lagrange multipliers. Then we have the following condition for optimality:

$$
\frac{\partial L}{\partial u_{i j k}}=\frac{1}{2} \frac{\left(a_{i k} p_{k j}\right)^{2}}{u_{i j k}^{2}}-\mu_{i j}=0,
$$

from which we have

$$
u_{i j k}=\frac{a_{i k} p_{k j}}{\sqrt{2 \mu_{i j}}}
$$

which is substituted into

$$
\frac{\partial L}{\partial \mu_{i j}}=\sum_{k=1}^{K} u_{i j k}-1=0
$$

to obtain

$$
\sqrt{2 \mu_{i j}}=\sum_{k=1}^{K} a_{i k} p_{k j}
$$

Substitution of (13) into (12) gives

$$
u_{i j k}=\frac{a_{i k} p_{k j}}{\sum_{l=1}^{K} a_{i l} p_{l j}} .
$$

Next, we solve the following optimality condition for $p_{k j}$ :

$$
\frac{\partial L}{\partial p_{k j}}=\frac{\partial F_{L B}}{\partial p_{k j}}=\sum_{i=1}^{m}\left(a_{i k} q_{i j}-\frac{a_{i k}^{2} p_{k j}}{u_{i j k}}\right)=0,
$$

and have

$$
p_{k j}=\frac{\sum_{i=1}^{m} a_{i k} q_{i j}}{\sum_{i=1}^{m} \frac{a_{i k}^{2}}{u_{i j k}}}
$$

into which we substitute (14) to have

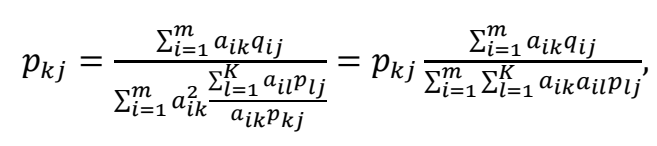

from which we have an iterative formula for $p_{k j}$ as follows:

$$
p_{k j}^{(t+1)}=p_{k j}^{(t)} \frac{\sum_{i=1}^{m} a_{i k} q_{i j}}{\sum_{i=1}^{m} \Sigma_{l=1}^{K} a_{i k} a_{i l} p_{l k}^{(t)}}
$$

where $t$ denotes the number of iterations for $t=0,1, \ldots, T$ with the maximum iteration number $T$. The above formula (15) can be written in a matrix form as

$$
P^{(t+1)}=P^{(t)} \odot\left(A^{T} Q\right) \oslash\left(A^{T} A P^{(t)}\right),
$$


where $\odot$ and $\oslash$ denote the element-wise multiplication and division, respectively, because $p_{k j}^{(t)}, \quad \sum_{i=1}^{m} a_{i k} q_{i j}$ and $\sum_{i=1}^{m} \sum_{l=1}^{K} a_{i k} a_{i l} p_{l k}^{(t)}$ in $(15)$ are the $(k, j)$ elements of matrices $P^{(t)}, A^{T} Q$ and $A^{T} A P^{(t)}$ in (16), respectively.

Proposition 1 The sequence $\left\{E\left(P^{(t)}\right)\right\}_{t=0,1, \ldots}$ converges.

Proof. Let

$$
u_{i j k}^{(t+1)}=\frac{a_{i k} p_{k j}^{(t)}}{\sum_{l=1}^{K} a_{i l} p_{l j}^{(t)}}
$$

be an instance of $u_{i j k}$ substituted $p_{k j}^{(t)}$ for $p_{k j}$ in (14). Then (15) can be written as

$$
p_{k j}^{(t+1)}=\frac{\sum_{i=1}^{m} a_{i k} q_{i j}}{\sum_{i=1}^{m} \frac{a_{i k}^{2}}{u_{i j k}^{(t+1)}}}
$$

Since (17) is the solution to $\partial L / \partial u_{i j k}=0$ and $\partial L / \partial \mu_{i j}=0$ with $P=P^{(t)}$, we find that $F_{L B}\left(P^{(t)}, U^{(t+1)}\right) \geq F_{L B}\left(P^{(t)}, U^{(t)}\right)$. Similarly, since (18) is the solution to $\partial L / \partial p_{k j}=0$ with $U=$ $U^{(t+1)}$, we find that $F_{L B}\left(P^{(t+1)}, U^{(t+1)}\right) \geq F_{L B}\left(P^{(t)}, U^{(t+1)}\right)$, i.e.,

$$
F_{L B}\left(P^{(t+1)}, U^{(t+1)}\right) \geq F_{L B}\left(P^{(t)}, U^{(t)}\right)
$$

Next, substituting (14) into (9), we have

$$
\begin{gathered}
F_{L B}(P, U)=\sum_{i=1}^{m} \sum_{j=1}^{n}\left[q_{i j} \sum_{k=1}^{K} a_{i k} p_{k j}-\frac{1}{2} \sum_{k=1}^{K} \frac{\left(a_{i k} p_{k j}\right)^{2}}{a_{i k} p_{k j}} \sum_{l=1}^{K} a_{i l} p_{l j}\right] \\
=\sum_{i=1}^{m} \sum_{j=1}^{n}\left[q_{i j} \sum_{k=1}^{K} a_{i k} p_{k j}-\frac{1}{2}\left(\sum_{k=1}^{K} a_{i k} p_{k j}\right)^{2}\right]=F(P),
\end{gathered}
$$

from which and (19) we have

$$
F\left(P^{(t+1)}\right) \geq F\left(P^{(t)}\right) .
$$

Considering the relationship between $E(P)$ and $F(P)$ given by $E(P)=\sum_{i=1}^{m} \sum_{j=1}^{n} q_{i j}^{2}-2 F(P)$, we have the following inequality from (20):

$$
E\left(P^{(t+1)}\right) \leq E\left(P^{(t)}\right)
$$

that is, the sequence $\left\{E\left(P^{(t)}\right)\right\}_{t=0,1, \ldots}$ is monotonically nonincreasing, and is also bounded below by 0 , i.e., $E(P) \geq 0$ by definition. Consequently, the sequence $\left\{E\left(P^{(t)}\right)\right\}_{t=0,1, \ldots}$ converges to a number greater than or equal to 0 .

\section{Color Reproduction}

Let $\bar{x}(\lambda), \bar{y}(\lambda)$ and $\bar{z}(\lambda)$ be the color matching functions for the $19312^{\circ} \mathrm{CIE}$ standard colorimetric observer [], where $\lambda$ denotes a wavelength, and let $\bar{S}(\lambda)$ be the relative spectral power distribution of an illuminant. Then the CIE XYZ tristimulus values of a spectral reflectance $\bar{p}(\lambda)$ are given by

$$
\begin{aligned}
& \bar{X}=\bar{k} \int \bar{s}(\lambda) \bar{p}(\lambda) \bar{x}(\lambda) d \lambda, \\
& \bar{Y}=\bar{k} \int \bar{s}(\lambda) \bar{p}(\lambda) \bar{y}(\lambda) d \lambda, \\
& \bar{Z}=\bar{k} \int \bar{s}(\lambda) \bar{p}(\lambda) \bar{z}(\lambda) d \lambda,
\end{aligned}
$$

where $\bar{k}$ is a normalizing factor given by $\bar{k}=\int \bar{s}(\lambda) \bar{y}(\lambda) d \lambda$. We use the trapezoidal rule for computing the above integrals. The transformation from the CIE XYZ tristimulus values into the standard RGB (sRGB) is as follows. First, we compute the linear transformation

$$
\left[\begin{array}{l}
R \\
G \\
B
\end{array}\right]=\left[\begin{array}{ccc}
3.2406 & -1.5372 & -0.4986 \\
-0.9689 & 1.8758 & 0.0415 \\
0.0557 & -0.2040 & 1.0570
\end{array}\right]\left[\begin{array}{c}
\bar{X} \\
\bar{Y} \\
\bar{Z}
\end{array}\right]
$$

where note that similar but slightly different transformation matrices are also proposed and used in practice.

Next, a nonlinear luminance encoding, which is also known as gamma encoding, is calculated to obtain sRGB values as follows:

$$
\begin{aligned}
& R_{\mathrm{SRGB}}=\left\{\begin{array}{cl}
1.055 R^{\frac{1}{2.4}}-0.055, & \text { if } R>0.0031308, \\
12.92 R, & \text { if } R \leq 0.0031308 ;
\end{array}\right. \\
& G_{\mathrm{SRGB}}=\left\{\begin{array}{cl}
1.055 G^{\frac{1}{2.4}}-0.055, & \text { if } G>0.0031308, \\
12.92 G, & \text { if } G \leq 0.0031308 ;
\end{array}\right. \\
& B_{\mathrm{SRGB}}=\left\{\begin{array}{cl}
1.055 B^{\frac{1}{2.4}}-0.055, & \text { if } B>0.0031308, \\
12.92 B, & \text { if } B \leq 0.0031308 .
\end{array}\right.
\end{aligned}
$$

Consequently, we obtain the sRGB values $\left(R_{\mathrm{sRGB}}, G_{\mathrm{sRGB}}, B_{\mathrm{sRGB}}\right)$ transformed from the CIE XYZ tristimulus values $(\bar{X}, \bar{Y}, \bar{Z})$.

\section{Experimental Results}

In this section, we show experimental results of spectral reflectance estimation and color reproduction. Figure 1 shows the Macbeth ColorChecker (MCC), of which the manufacturer's sRGB D65 color values are also given on the Internet (ColorChecker, Wikipedia. URL: https://en.wikipedia.org/wiki/ ColorChecker). Although the 24 squares of painted samples in MCC are not materials but colorants, the proposed method can handle both of them equally because the Neugebauer model is valid for general colorimetric data as shown in Subsection 2.1. 


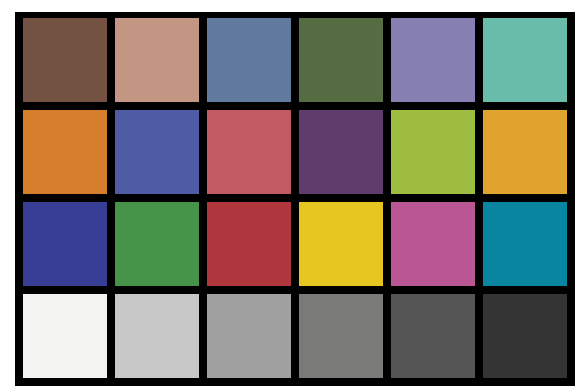

Figure 1 Macbeth ColorChecker.

The spectral reflectance data of the 24 colors in MCC are shown in Figure 2, where the line colors correspond to the MCC colors. These data are provided by the Munsell Color Science Laboratory (URL: http://www.cis.rit.edu/research/mcsl2/online/cie.php).

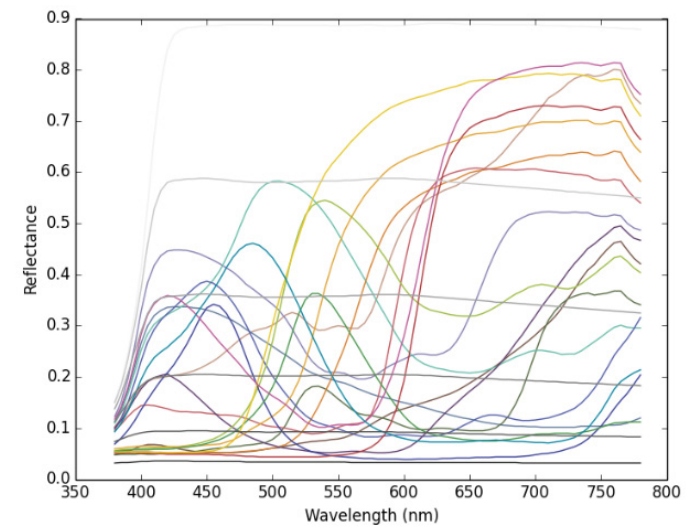

Figure 2 Reflectance spectra of Macbeth ColorChecker colors.

In the following experiments, we store the above data in a matrix $Q \in \mathbb{R}^{m \times n}$ where $m$ denotes the number of colors in MCC, i.e., $m=24$, and $n$ denotes the number of spectral data in which the wavelengths between $380 \mathrm{~nm}$ (nanometer) and $780 \mathrm{~nm}$ are sampled at $5 \mathrm{~nm}$ intervals, i.e., $n=81$.

Figure 3 shows the change in the value of the objective function $E(P)$ of the proposed method with the Neugebauer model described in Section 2.1, where the vertical and horizontal axes denote the objective function value and the number of iterations $t$ with the maximum iteration number $T=50$, respectively. The objective function value monotonically decreases with the increase in the number of iterations, that supports the claim in Proposition 1 experimentally.

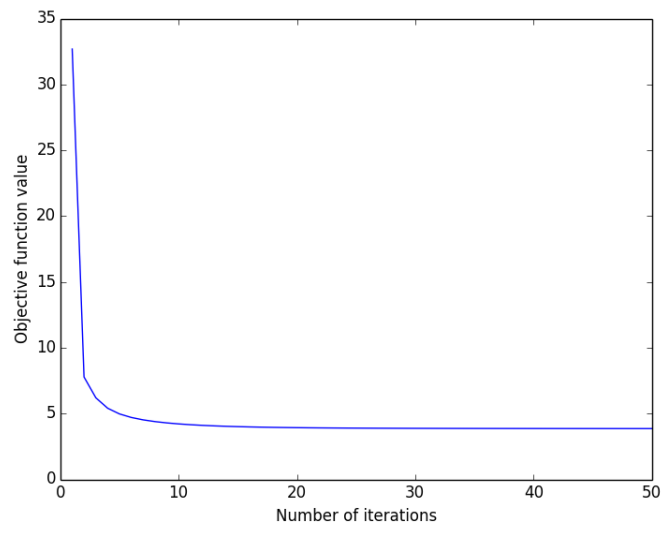

Figure 3 Change in objective function value.
Figure 4 shows the estimated spectral reflectance of the Neugebauer primaries by the sequential coordinate-wise algorithm (SCA) $[6,8]$, where the vertical and horizontal axes denote the reflectance and wavelength, respectively, and the colors of solid lines correspond to the primary colors, and the broken line denotes white color. On the other hand, Figure 5 shows the estimated spectral reflectance by the proposed method with the Neugebauer model, and is similar to Figure 4, although the algorithms are different from each other. We measured CPU time for estimating the spectral reflectance on an Intel Core-i3-equipped PC using Portable Python 2.7.6.1. Then SCA and the proposed method required 1.12 and 0.028 seconds, respectively, from which we conclude that the proposed method is computationally more efficient than SCA.

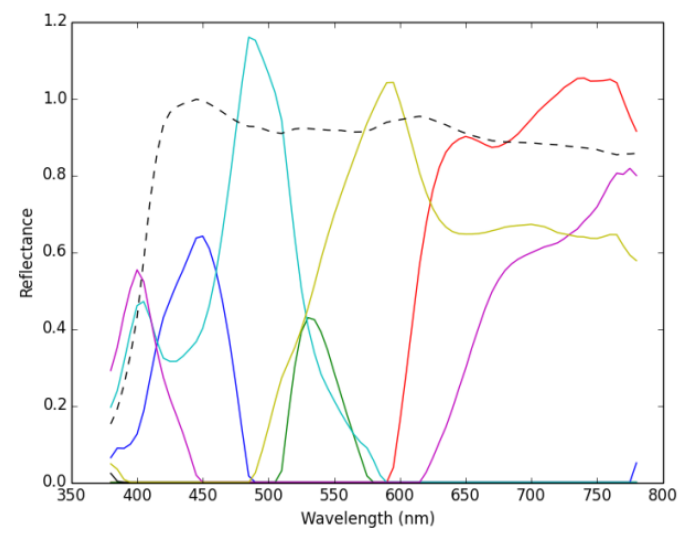

Figure 4 Estimated spectral reflectance by SCA $[6,8]$.

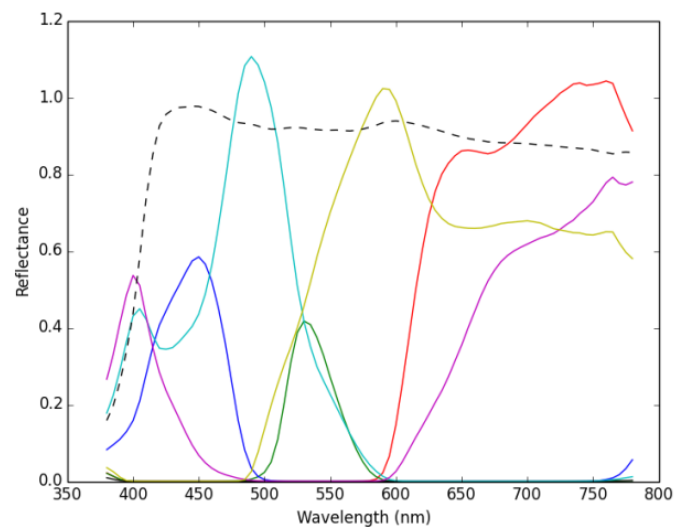

Figure 5 Estimated spectral reflectance by the proposed method with Neugebauer model.

Next, we compare sparse Neugebauer model with nonsparse or conventional Neugebauer model in the proposed method. Figure 6 shows the coefficients in two Neugebauer models for the upper left color in MCC in Figure 1, where the vertical and horizontal axes denote the value of each coefficient and the Neugebauer primaries, respectively. the blue and red bars denote conventional (nonsparse) and sparse Neugebauer models, respectively. In the conventional model (blue bars), all Neugebauer primaries have positive values. On the other hand, in sparse one (red bars), only four colors (R, G, $\mathrm{B}$ and $\mathrm{M}$ ) have positive values, and the other coefficients are strictly zero. 


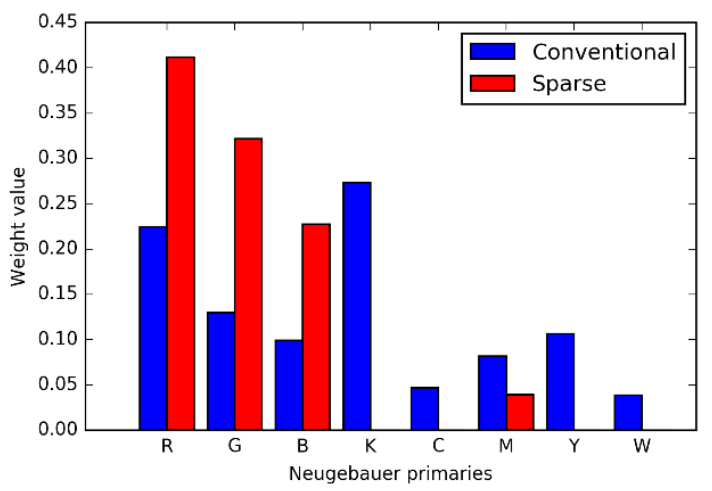

Figure 6 Coefficients in Neugebauer models.

Figure 7 shows the estimated spectral reflectance of the Neugebauer primaries by the proposed method with the sparse Neugebauer model. Each curve in Figure 7 has greater variance than that in Figure 5 or 4.

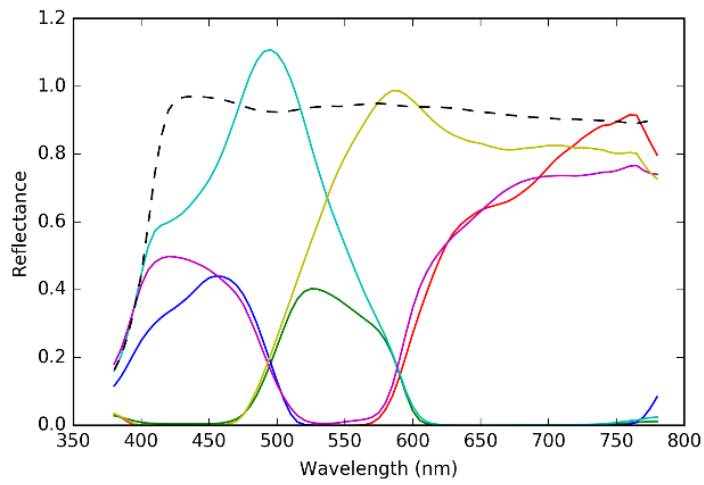

Figure 7 Estimated spectral reflectance by the proposed method with sparse Neugebauer model.

From Figure 8 to Figure 11, we show the reflectance spectra of the colors in MCC from the first to fourth rows, respectively (here we divide the $24 \mathrm{MCC}$ colors into 4 figures each of which includes the spectral reflectance curves of 6 colors for visibility), where solid lines show the original reflectance spectra, and broken lines show their estimated ones by the proposed method with the Neugebauer model.

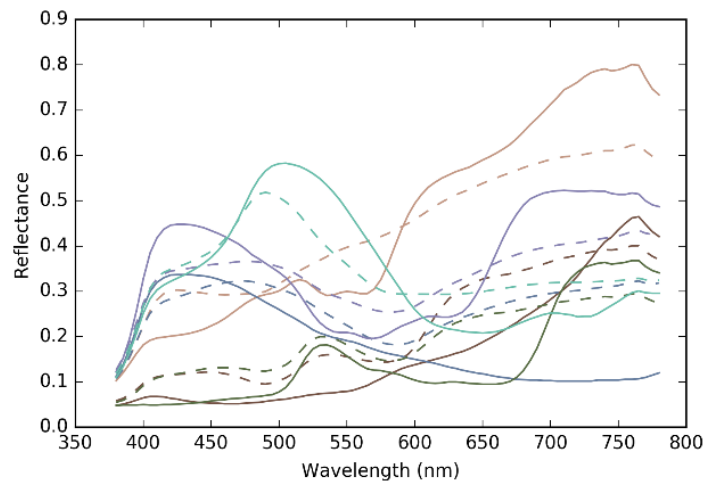

Figure 8 The original reflectance spectra of the colors in the first row of MCC (solid lines) and their estimated ones by the proposed method with Neugebauer model (broken lines).

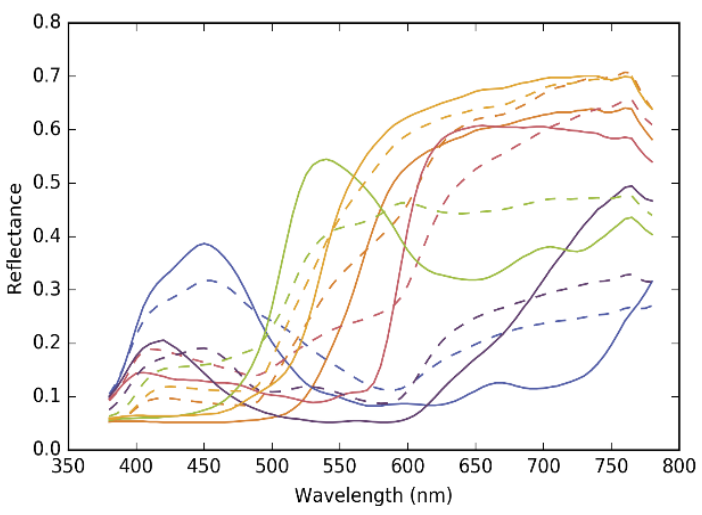

Figure 9 The original reflectance spectra of the colors in the second row of MCC (solid lines) and their estimated ones by the proposed method with Neugebauer model (broken lines).

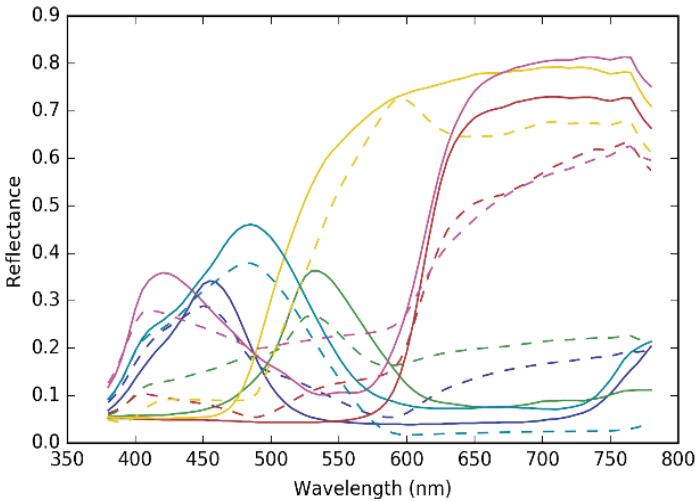

Figure 10 The original reflectance spectra of the colors in the third row of MCC (solid lines) and their estimated ones by the proposed method with Neugebauer model (broken lines).

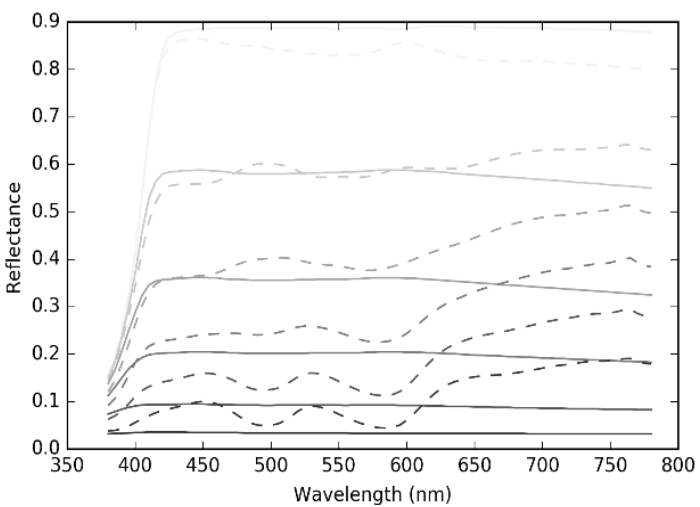

Figure 11 The original reflectance spectra of the colors in the fourth row of MCC (solid lines) and their estimated ones by the proposed method with Neugebauer model (broken lines).

On the other hand, from Figure 12 to Figure 15, we show the estimated reflectance spectra by the proposed method with sparse Neugebauer model, as well as Figures 8-11 with the Neugebauer model. 


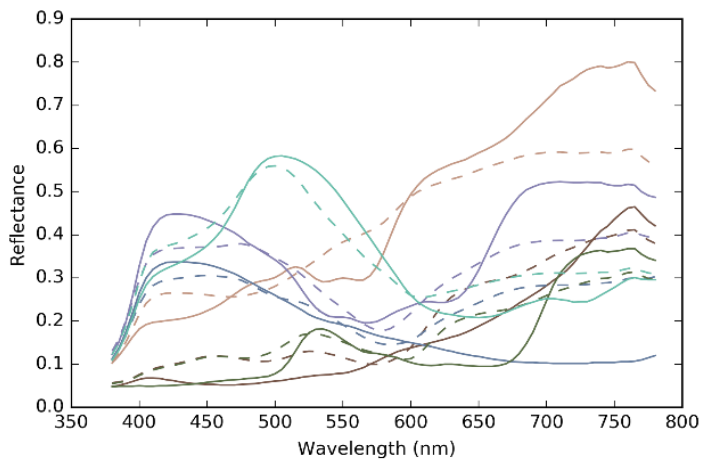

Figure 12 The original reflectance spectra of the colors in the first row of MCC (solid lines) and their estimated ones by the proposed method with sparse Neugebauer model (broken lines).

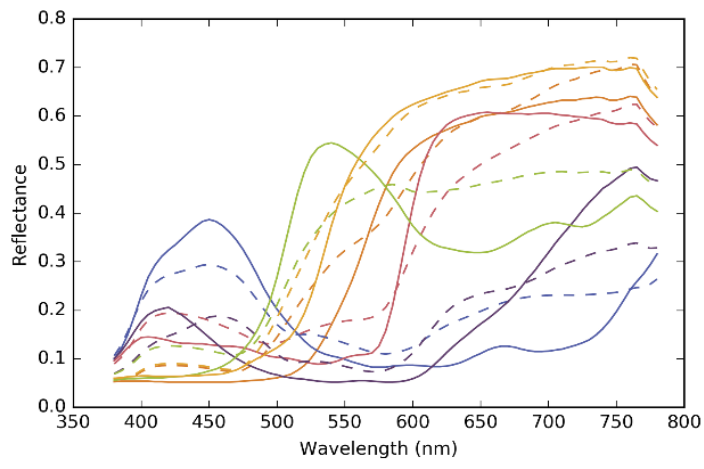

Figure 13 The original reflectance spectra of the colors in the second row of MCC (solid lines) and their estimated ones by the proposed method with sparse Neugebauer model (broken lines).

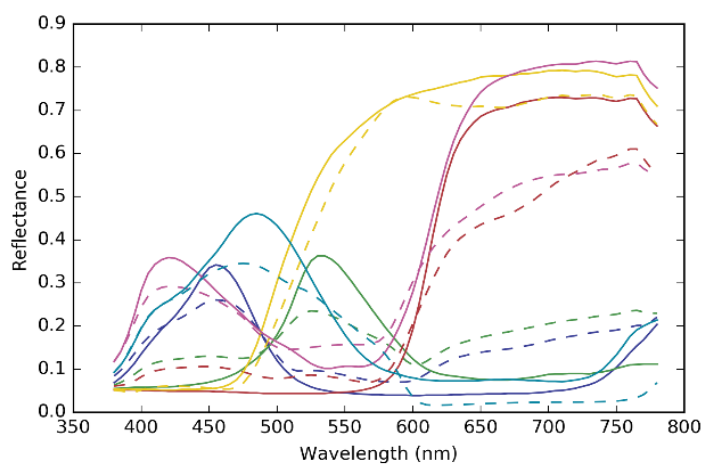

Figure 14 The original reflectance spectra of the colors in the third row of MCC (solid lines) and their estimated ones by the proposed method with sparse Neugebauer model (broken lines).

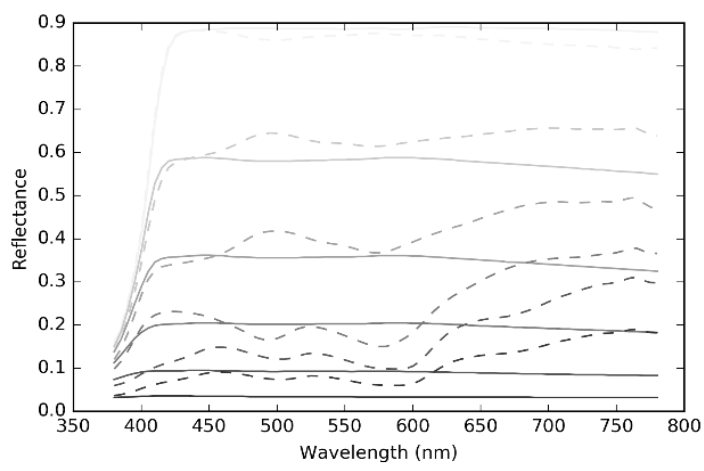

Figure 15 The original reflectance spectra of the colors in the fourth row of MCC (solid lines) and their estimated ones by the proposed method with sparse Neugebauer model (broken lines)
We evaluate the mean squared error between the original and estimated reflectance spectra given by

$$
\operatorname{MSE}_{i}=\frac{1}{n} \sum_{j=1}^{n}\left(q_{i j}-\sum_{k=1}^{K} a_{i k} p_{k j}\right)^{2}
$$

for the $i$ th color in MCC. Figure 16 shows the result where the vertical and horizontal axes denote the mean squared error and the identification number of MCC colors, respectively, and the blue and red bars denote the conventional nonsparse and the sparse Neugebauer models, respectively. In 18 colors among all 24 colors, the sparse Neugebauer model achieved smaller $\mathrm{MSE}_{i}$ than the conventional one. The average of $\mathrm{MSE}_{i}$ for $i=1, \ldots, m$ given by

$$
\mathrm{MSE}=\frac{1}{m} \sum_{i=1}^{m} \mathrm{MSE}_{i}
$$

are $7.66 \times 10^{-3}$ for the conventional Neugebauer model and $6.83 \times 10^{-3}$ for the sparse one.

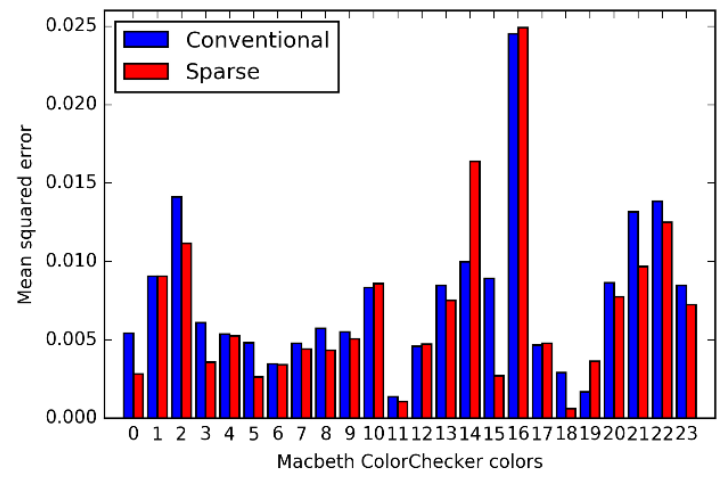

Figure 16 Mean squared error of estimated reflectance spectra.

Next, we also evaluate the same measure using the leave-oneout cross-validation [11], where the spectral reflectance of the $i$ th color in $\mathrm{MCC}$ is removed from $Q$ to evaluate $\mathrm{MSE}_{i}$. Figure 17 shows the result, where the sparse Neugebauer model (red bars) achieved smaller $\mathrm{MSE}_{i}$ in 15 colors among all 24 colors than the conventional Neugebauer model (blue bars). The values of MSE are $1.37 \times 10^{-2}$ for the conventional Neugebauer model and $1.15 \times 10^{-2}$ for the sparse one.

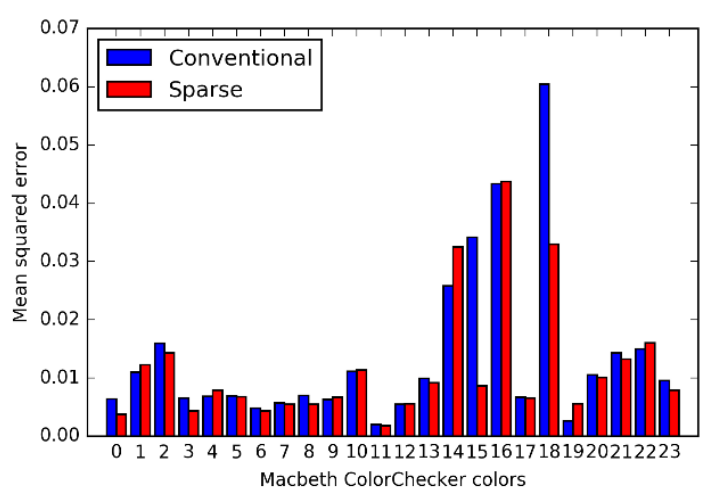

Figure 17 Mean squared error of estimated reflectance spectra evaluated by the leave-one-out cross-validation.

Finally, we show the results of color reproduction from the estimated reflectance spectra. Figure 18 shows the original Neugebauer primaries: red, green, blue, black, cyan, magenta, yellow and white. 


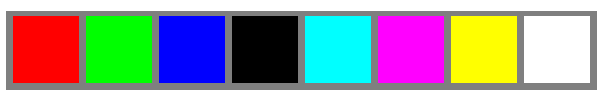

Figure 18 Neugebauer primaries.

Figure 19 shows the reproduced Neugebauer primaries with the conventional Neugebauer model, in which blue is wrongly transformed into magenta.

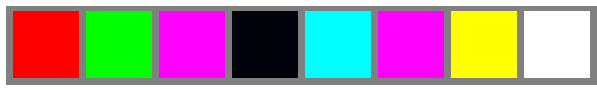

Figure 19 Reproduced Neugebauer primaries from the estimated reflectance spectra with Neugebauer model.

On the other hand, the reproduced Neugebauer primaries with the sparse Neugebauer model is shown in Figure 20, where blue is correctly reproduced as well as the other colors.

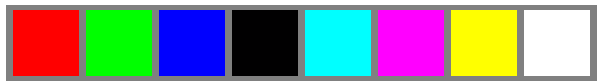

Figure 20 Reproduced Neugebauer primaries from the estimated reflectance spectra with sparse Neugebauer model.

Figures 21 and 22 show the reproduced MCC colors with the conventional and sparse Neugebauer models, respectively. The reproduced colors are similar to the original ones in Figure 1. Additionally, note that the proposed method can be applied to not only the CMYK-printed colors like MCC but also more general tristimulus values including material colors.

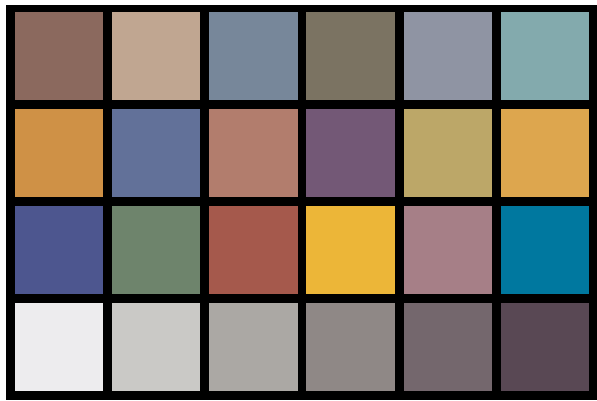

Figure 21 Reproduced MCC colors with Neugebauer model.

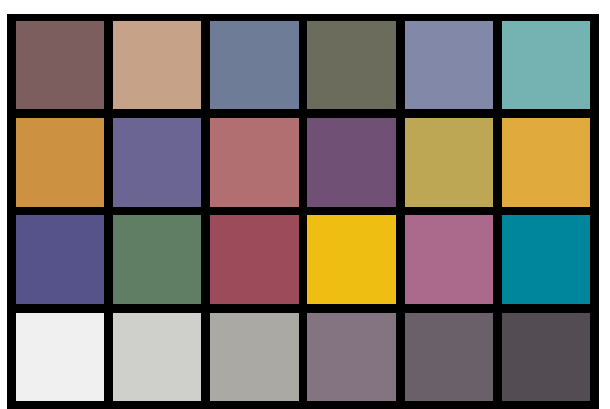

Figure 22 Reproduced MCC colors with sparse Neugebauer model.

We evaluate the mean absolute error between the original and reproduced $\mathrm{MCC}$ colors given by

$$
\operatorname{MAE}_{i}=\frac{\left|r_{i}-\tilde{r}_{i}\right|+\left|g_{i}-\tilde{g}_{i}\right|+\left|b_{i}-\tilde{b}_{i}\right|}{3}
$$

for the $i$ th color in MCC, where $\tilde{r}_{i}, \tilde{g}_{i}$ and $\tilde{b}_{i}$ denote the reproduced sRGB values from the estimated reflectance spectra. Figure 23 shows the result, where the sparse Neugebauer model (red bars) achieved smaller $\mathrm{MAE}_{i}$ in 20 colors among all 24 colors than the conventional Neugebauer model. The average of $\mathrm{MAE}_{i}$ for $i=$ $1, \ldots, m$ given by

$$
\mathrm{MAE}=\frac{1}{m} \sum_{i=1}^{m} \mathrm{MAE}_{i}
$$

are 17.65 for the conventional Neugebauer model and 13.81 for the sparse one.

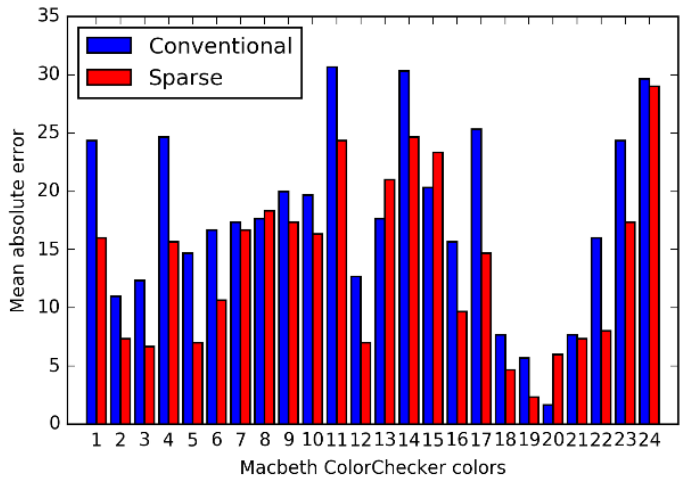

Figure 23 Mean absolute error of reproduced MCC colors.

Consequently, the sparse Neugebauer model improved the performance of both spectral reflectance estimation and color reproduction from the estimated spectral reflectance compared with the conventional Neugebauer model.

\section{Conclusion}

In this paper, we proposed a method for estimating spectral reflectance based on the Neugebauer model and its sparse version. The sparse Neugebauer model improved the estimation performance compared with the conventional Neugebauer model. We also proved the convergence property of the proposed algorithm for spectral reflectance estimation. Additionally, we reproduced the colors from the estimated spectral reflectance, in which the sparse Neugebauer model also improved the performance compared with the conventional one.

For future work we would like to improve the performance of both spectral reflectance estimation and color reproduction by robustifying the proposed method, and compare the proposed method with other related methods including learning-based methods. We are also planning an application of the proposed method to the design of spectral reflectance for controlled color reproduction under various illuminations.

\section{Conflict of Interest}

The authors declare no conflict of interest.

\section{Acknowledgment}

This work was supported by JSPS KAKENHI Grant Number JP16H03019.

\section{References}

[1] S. Westland, Computational Colour Science Using MATLAB, Wiley, 2004.

[2] G. Sharma, S. Wang, "Spectrum recovery from colorimetric data for color reproductions," in Proc. SPIE 4663, Color Imaging: Device-Independent Color, Color Hardcopy, and Applications VII, San Jose, CA, 2002. 
[3] T. Harifi, S. H. Amirshahi , F. Agahian, "Recovery of reflectance spectra from colorimetric data using principal component analysis embedded regression technique," Opt. Rev., 15(6), 302-308, 2008.

[4] M. M. Amiri, S. H. Amirshahi, "A step by step recovery of spectral data from colorimetric information,” J. Opt., 44(4), 373-383, 2015.

[5] G. Wu, X. Shen, Z. Liu, S. Yang, M. Zhu, "Reflectance spectra recovery from tristimulus values by extraction of color feature match," Opt. Quant. Electron., 48(1), 48:64, 2016.

[6] V. Franc, V. Hlaváč, M. Navara, "Sequential coordinate-wise algorithm for the non-negative least squares problem," in Gagalowicz A., Philips W. (eds) Computer Analysis of Images and Patterns. CAIP 2005. Lecture Notes in Computer Science, vol 3691. Springer, Berlin, Heidelberg, 2005.

[7] E. Reinhard, E. A. Khan, A. O. Akyuz, G. Johnson, Color Imaging: Fundamentals and Applications, A K Peters/CRC Press, 2008.

[8] K. Inoue, K. Hara, K. Urahama, "Reflectance spectra recovery with nonnegativity constraints," in 2016 International Symposium on Intelligent Signal Processing and Communication Systems, Phuket, Thailand, 2016.

[9] D. Shaked, N. Arad, , A. Fitzhugh, I. Sobel, "Color diffusion: Error-diffusion for color halftones,” HP Labs Technical Reports, HPL-96-128R1, 1999.

[10] S. Boyd, L. Vandenberghe, Convex Optimization, Cambridge University Press, 2004.

[11] S. Arlot, A. Celisse, "A survey of cross-validation procedures for model selection," Statistics Surveys, 4, 40-79, 2010. 